\title{
Post-Processing Functions for a Biased Physical Random Number Generator
}

\author{
Patrick Lacharme \\ Imath, Université de Toulon
}

\begin{abstract}
A corrector is used to reduce or eliminate statistical weakness of a physical random number generator. A description of linear corrector generalizing post-processing described by M. Dichtl at FSE'07 [5] is introduced. A general formula for non linear corrector, determining the bias and the minimal entropy of the output of a function is given. Finally, a concrete and efficient construction of post-processing function, using resilient functions and cyclic codes, is proposed.
\end{abstract}

Keywords: bias, linear correcting codes, Fourier transform, resilient functions, entropy.

\section{Introduction}

The scheme of a true random number generator consists of two different parts. The first one is a noise source using a physical non deterministic phenomenon producing a raw binary sequence. The second one is a corrector compressing this sequence in order to provide randomness extraction. 1 At FSE'07, M. Dichtl proposed several true random number generators designed to reduce the bias of the noise source and extract more entropy than known algorithms [5]. He considered that the physical source produces statistically independents bits with constant bias. In his conclusion, the author suggested to extend his work in many directions : compression rates, other input sizes and systematic construction of good post-processing functions.

In this paper, we study the output bias of a function. The same assumptions as in [5] are taken : the input bits of the function are independents and have the same bias. General constructions of functions achieving very good output bias are exposed. Furthermore, these functions are very efficiently implemented in smart-card applications. The output bias of a linear corrector is bounded in Section 2, using linear correcting codes. Section 3 presents the explicit calculation of the output bias of a function with its Fourier transform. Resilient functions are used in Section 4 to construct correctors and Section 5 proposes an estimation of minimal entropy of the output sequence.

\footnotetext{
${ }^{1}$ True random number generator should not be used for cryptographic purposes without a more complex structure as a pseudo random generator 3 .
} 


\section{A Linear Corrector}

We consider a physical noise source providing a raw binary sequence. The bits $x_{i}$ are independent and display a constant bias $e$, defined by

$$
e=\frac{1}{2}\left(P\left(x_{i}=1\right)-P\left(x_{i}=0\right)\right),
$$

with $P\left(x_{i}=1\right)=\frac{1}{2}+e$ and $P\left(x_{i}=0\right)=\frac{1}{2}-e$. This assumption is taken in order to get a simple formula and to compare our correctors with the correctors proposed in [5] on the same hypothesis. Nevertheless Theorem 1 can be generalized with non constant bias assumption.

The linear corrector $H$ proposed in [5, maps 16 bits to 8 bits. For $x=$ $\left(x_{0}, \ldots, x_{15}\right)$ the input vector and $y=\left(y_{0}, \ldots, y_{7}\right)$ the output vector, the corrector $H$ is defined by the following relation

$$
\forall i=0, \ldots, 7 \quad y_{i}=x_{i}+x_{i+1 \bmod 8}+x_{i+8} \bmod 2 .
$$

The compression rate of $H$ is 2 , exactly the same rate as the xor corrector

$$
y_{i}=x_{2 i}+x_{2 i+1} \bmod 2 .
$$

If we note $X_{1}$ and $X_{2}$ the two input bytes of $H,+$ the bitwise xor and $R L(X, i)$ the circular rotation of $i$ bits, we can write $H$ in pseudocode

$$
H\left(X_{1}, X_{2}\right)=X_{1}+\operatorname{RL}\left(X_{1}, 1\right)+X_{2} .
$$

Two furthers improvements of $H$ are presented

$$
\begin{aligned}
& H_{2}\left(X_{1}, X_{2}\right)=X_{1}+\operatorname{RL}\left(X_{1}, 1\right)+\operatorname{RL}\left(X_{1}, 2\right)+X_{2}, \\
& H_{3}\left(X_{1}, X_{2}\right)=X_{1}+\operatorname{RL}\left(X_{1}, 1\right)+\operatorname{RL}\left(X_{1}, 2\right)+\operatorname{RL}\left(X_{1}, 4\right)+X_{2} .
\end{aligned}
$$

The author says that if the bias of any input bits is $e$, then the lowest power of $e$ in the bias of output bytes is 3 for $H, 4$ for $H_{2}$ and 5 for $H_{3}$. His approach is to determine probability of every inputs, and to sum up the probability for all input leading to the same output of the corrector.

A simple mathematical proof of previous results is determined using the matricial representation of a linear corrector. For $x=\left(x_{1}, \ldots, x_{n}\right)$ and $y=$ $\left(y_{1}, \ldots, y_{m}\right)$, any linear binary corrector mapping $n$ bits to $m$ bits, is defined as the product of the vector $x$ by the binary matrix $G=\left(g_{i, j}\right)$ :

$$
\left(\begin{array}{l}
g_{1,1} \ldots g_{1, n} \\
\vdots \\
g_{m, 1} \ldots g_{m, n}
\end{array}\right)\left(\begin{array}{l}
x_{1} \\
\vdots \\
x_{n}
\end{array}\right)=\left(\begin{array}{l}
y_{1} \\
\vdots \\
y_{m}
\end{array}\right)
$$

Theorem 1. Let $G$ be a linear corrector mapping $n$ bits to $m$ bits. Then the bias of any non zero linear combination of the output bits is less or equal than $2^{d-1} e^{d}$, where $d$ is the minimal distance of the linear code constructed by the generator matrix $G$. 
Proof. Firstly, recall that if $n$ bits $x_{1}, \ldots, x_{n}$ have a bias $e$, then the bias of $x_{1}+\ldots+x_{n} \bmod 2$ is $2^{n-1} e^{n}$ (the proof is a simple induction) [7].

The matrix $G$ is seen as a generator matrix of a $[n, k, d]$ linear code. By definition of the minimal distance of the code, any non zero linear combination of output bits is the sum of, at least, $d$ input bits. We conclude that the bias of any non zero linear combination of output bits is less or equal than $2^{d-1} e^{d}$.

This theorem gives an upper bound of the output bias for an arbitrary linear corrector. In particular, the matrix corresponding to $H, H_{2}$ and $H_{3}$ are respectively generator matrix of $[16,8,3],[16,8,4]$ and $[16,8,5]$ linear codes. Then the bias of any linear combination of output bits is bounded, respectively by $4 e^{3}$, $8 e^{4}$ and $16 e^{5}$. Theorem 5 of Section 5 allows to conclude on Dichtl results on the lowest power of $e$ in the output bytes bias.

Any linear $[n, m, d]$-code provides a linear corrector with an estimation of its output bias. The compression rate of a corrector mapping $n$ bits to $m$ bits is defined by $n / m$. A table of linear codes gives good linear corrector with variable compression rates and input sizes [6]. The hardware implementation of linear corrector is efficiently achieved as a simple multiplication of an input vector by a constant matrix. A cyclic code provides a more compact implementation of the corrector and improves its realisation.

There are no linear binary codes of length 16 , dimension 8 with minimal distance greater than 5 [6]. In theses conditions, to minimize output bias, we must search non linear correctors.

\section{Non Linear Corrector}

Let $f$ be a corrector mapping $n$-bits to $m$-bits. A non zero linear combination of output bits of $f$ is defined using a $m$-bits vector $u \neq 0$, by the Boolean function $\phi_{u}(x)=\sum_{i=1}^{m} u_{i} f_{i}(x)=u . f(x)$. For an input bits bias $e$, the bias of this linear combination is

$$
\Delta_{u}=\frac{1}{2}\left(P\left(\phi_{u}(x)=1\right)-P\left(\phi_{u}(x)=0\right)\right) .
$$

The bias $\Delta_{u}$ can be directly computed using the truth table of $\phi_{u}(x)$ and the input bias $e$ by the formula

$$
\begin{aligned}
2 \Delta_{u}(e) & =\sum_{\substack{x \in \mathbf{F}_{2}^{n} \\
\phi_{u}(x)=1}}\left(\frac{1}{2}-e\right)^{n-w_{h}(x)}\left(\frac{1}{2}+e\right)^{w_{h}(x)}-\sum_{\substack{x \in \mathbf{F}_{2}^{n} \\
\phi_{u}(x)=0}}\left(\frac{1}{2}-e\right)^{n-w_{h}(x)}\left(\frac{1}{2}+e\right)^{w_{h}(x)} \\
& =\sum_{x \in \mathbf{F}_{2}^{n}}\left(\frac{1}{2}-e\right)^{n-w_{h}(x)}\left(\frac{1}{2}+e\right)^{w_{h}(x)}(-1)^{\phi_{u}(x)+1} .
\end{aligned}
$$

Therefore

$$
\Delta_{u}(e)=-\frac{1}{2} \sum_{x \in \mathbf{F}_{2}^{n}}\left(\frac{1}{2}-e\right)^{n-w_{h}(x)}\left(\frac{1}{2}+e\right)^{w_{h}(x)}(-1)^{\phi_{u}(x)} .
$$


For a Boolean function $f$, the Hamming weight $w_{H}(f)$ denotes the number of ' 1 ' in its truth table. The Walsh transform of $f$ is :

$$
\forall v \in \mathbf{F}_{2}^{n} \quad \widehat{f}(v)=\sum_{x \in \mathbf{F}_{2}^{n}}(-1)^{f(x)+v \cdot x} .
$$

Lemma 1. Let $x$ be a binary vector on $\mathbf{F}_{2}^{n}$ such that the bits $x_{i}$ are independent. Then

$$
\sum_{a \in \mathbf{F}_{2}^{n}} P(x=a)(-1)^{v \cdot a}=(-2 e)^{w_{H}(v)} .
$$

Proof. By independency of the bits $x_{i}$,

$$
\begin{aligned}
\sum_{a \in \mathbf{F}_{2}^{n}} P(x=a)(-1)^{v \cdot a} & =\prod_{i=1}^{n} \sum_{a_{i}=0}^{1} P\left(x_{i}=a_{i}\right)(-1)^{v_{i} a_{i}} \\
& =\prod_{\substack{i=1 \\
v_{i}=1}}^{n}\left(P\left(x_{i}=0\right)-P\left(x_{i}=1\right)\right) \prod_{\substack{i=1 \\
v_{i}=0}}^{n}\left(P\left(x_{i}=0\right)+P\left(x_{i}=1\right)\right) \\
& =(-2 e)^{w_{H}(v) .}
\end{aligned}
$$

Theorem 2 presents a complete description of the bias of any non zero linear combination $\phi_{u}(x)=u . f(x)$ of a vectorial function $f$ relatively to the the input bias $e$ and the coefficients of the Walsh transform of $\phi_{u}$ :

Theorem 2. Let $f$ be a function which maps $n$ bits to $m$ bits and $e$ the input bit bias. Then the bias $\Delta_{u}(e)$ is

$$
\Delta_{u}(e)=\frac{1}{2^{n+1}} \sum_{v \in \mathbf{F}_{2}^{n}}(2 e)^{w_{H}(v)}(-1)^{w_{H}(v)+1} \widehat{\phi_{u}}(v) .
$$

Proof. By definition on bias $\Delta_{u}$,

$$
\begin{aligned}
2 \Delta_{u}(e) & =P\left(\phi_{u}(x)=1\right)-P\left(\phi_{u}(x)=0\right) \\
& =-\sum_{a \in \mathbf{F}_{2}^{n}} P(x=a)(-1)^{\phi_{u}(a)} .
\end{aligned}
$$

Moreover,

$$
\sum_{v \in \mathbf{F}_{2}^{n}}(-1)^{v \cdot(a+z)}=\left\{\begin{array}{l}
0 \text { for } a \neq z \\
2^{n} \text { for } a=z
\end{array}\right.
$$

Using equation (3) we get

$$
\sum_{a \in \mathbf{F}_{2}^{n}} P(x=a)(-1)^{\phi_{u}(a)}=2^{-n} \sum_{v \in \mathbf{F}_{2}^{n}} \sum_{a \in \mathbf{F}_{2}^{n}}(-1)^{v \cdot a} P(x=a) \sum_{z \in \mathbf{F}_{2}^{n}}(-1)^{\phi_{u}(z)+v \cdot z} .
$$

Therefore with Lemma 1 and definition of $\widehat{\phi_{u}}$,

$$
\Delta_{u}(e)=\frac{1}{2^{n+1}} \sum_{v \in \mathbf{F}_{2}^{n}}(2 e)^{w_{H}(v)}(-1)^{w_{H}(v)+1} \widehat{\phi_{u}}(v) .
$$


For example, let $f$ be the quadratic Boolean function defined by

$$
f(x)=f\left(x_{1}, x_{2}, x_{3}\right)=x_{2}+x_{3}+x_{1} x_{2}+x_{2} x_{3} \bmod 2,
$$

where the truth table and the Walsh coefficients are

\begin{tabular}{|c|c|c|}
\hline$x$ & $f(x)$ & $\hat{f}(x)$ \\
\hline 000 & 0 & 0 \\
001 & 1 & 4 \\
010 & 1 & 0 \\
100 & 0 & -4 \\
011 & 1 & 4 \\
101 & 1 & 0 \\
110 & 0 & 4 \\
111 & 0 & 0 \\
\hline
\end{tabular}

The probability $P(f(x)=0)=\frac{1}{2}-e$, computed using the truth table of $f$ :

$$
\begin{aligned}
P(f(x)=0) & =\left(\frac{1}{2}-e\right)^{3}+\left(\frac{1}{2}-e\right)^{2}\left(\frac{1}{2}+e\right)+\left(\frac{1}{2}-e\right)\left(\frac{1}{2}+e\right)^{2}+\left(\frac{1}{2}+e\right)^{3} \\
& =\frac{1}{2}+2 e^{2} .
\end{aligned}
$$

The output bias computed with Theorem 2 gives (with $u=1$ ) :

$$
\begin{gathered}
\Delta_{1}(e)=\frac{1}{16}(\hat{f}(000)+2 e \hat{f}(001)+2 e \hat{f}(010)+2 e \hat{f}(100) \\
\left.-4 e^{2} \hat{f}(011)-4 e^{2} \hat{f}(101)-4 e^{2} \hat{f}(110)+8 e^{3} \hat{f}(111)\right)
\end{gathered}
$$

After reduction, we get

$$
\Delta_{1}(e)=-2 e^{2} .
$$

Definition 1. Let $P$ be a polynomial of degree $d$, defined by

$$
P(X)=\sum_{i=0}^{d} a_{i} X^{i} .
$$

The valuation of $P$ is the minimal $i>0$ such that $a_{i} \neq 0$.

Corollary 1 is a consequence of Theorem 2 :

Corollary 1. Let $f$ be a function mapping $n$ bits to $m$ bits and $e$ the input bias of the function. For any vector $u$, we define for all $w$, with $0 \leq w \leq n$

$$
B_{w}=\sum_{\substack{v \in \mathbf{F}_{2}^{n} \\ w_{H}(v)=w}} \widehat{\phi_{u}}(v) .
$$

Then the bias of $\phi_{u}(x)$ is a polynomial of valuation $W$, with

$$
W=\min \left\{w \mid B_{w} \neq 0\right\} .
$$


Formula (2) gives a complete description of the bias and coefficients of the polynomial $\Delta_{u}(e)$ are determined by $B_{w}$.

In particular, if we consider the linear Boolean function which is the sum of $d$ variables, then $B_{w}=0$ for all $w \neq d$.

\section{A Resilient Corrector}

A $(n, m, t)$-resilient function is a function mapping $n$ bits to $m$ bits such that if $t$ input bits are fixed, there is no influence on the output :

Definition 2. 4] $A(n, m, t)$-resilient function is a function $f$ mapping $\mathbf{F}_{2}^{n}$ to $\mathbf{F}_{2}^{m}$ such that for any coordinates $i_{1}, \ldots i_{t}$ and for any binary constant $c_{1}, \ldots, c_{t}$ and for all $y \in \mathbf{F}_{2}^{m}$, we have

$$
P\left(f(x)=y \mid x_{i_{1}}=c_{1}, \ldots, x_{i_{t}}=c_{t}\right)=2^{-m},
$$

where $x_{i}$ with $i \notin\left\{i_{1}, \ldots, i_{t}\right\}$ verify $P\left(x_{i}=1\right)=P\left(x_{i}=0\right)=0.5$.

A $(n, m, t)$-linear resilient function is a linear corrector 2 and Theorem 3 shows the relation between resilience degree of a linear function and output bias :

Lemma 2. 4] $A(n \times m)$ binary matrix $M$ is a generator matrix of a linear $[n, m, d]$-code if and only if the function

$$
x \mapsto M^{t} x
$$

is a linear $(n, m, d-1)$-resilient function.

Theorem 3. Let $f$ be a linear $(n, m, t)$-resilient function. Then the bias of any non zero linear combination of the output bits is less or equal than $2^{t} e^{t+1}$.

Proof. From Lemma 2, any linear $(n, m, t)$-resilient function provides a generator matrix of a $[n, m, t+1]$-linear code. The theorem follows with Theorem 1 .

In the case of the $(n, m, t)$-resilient function is non linear, Theorem 4 evaluates the valuation of the output bias, using the resilience order of the function. Lemma 3 is known as xor Lemma [4:

Lemma 3. Let $f$ be a $(n, m, t)$-resilient function and $u$ a non zero vector in $\mathbf{F}_{2}^{m}$. Then, any non zero linear combination $u . f(x)$ of $f$ is a $(n, 1, t)$-resilient Boolean function.

G. Xiao and J. Massey propose a spectral characterization of $(n, m, t)$-resilient functions [1]:

Lemma 4. Let $f$ be a $(n, 1, t)$-resilient Boolean function. Then for all vector $v$ in $\mathbf{F}_{2}^{n}$ with $w_{H}(v) \leq t$, we have $\widehat{f}(v)=0$.

${ }^{2}$ In [9], Stinson, Martin and Sunar have proposed a true random number generator using a linear resilient function for the post-processing. 
Theorem 4. Let $f$ be $(n, m, t)$-resilient function and all input bits have a bias e. Then the bias of any non zero linear combination of output bits is a polynomial in $e$ of valuation greater than $t+1$.

Proof. Let $\phi_{u}(x)=u . f(x)$ be a linear combination of output bits. By Lemma 3 $\phi_{u}$ is a $(n, 1, t)$-resilient Boolean function. So, all Walsh coefficients $\widehat{\phi_{u}}(v)$ are null for all vector $v$ of Hamming weight less or equal than $t$ (Lemma 4). Using Theorem 2, we get

$$
\Delta_{u}(e)=\frac{1}{2^{n+1}} \sum_{\substack{v \in \mathbf{F}_{2}^{n} \\ w_{H}(v)>t}}(2 e)^{w_{H}(v)}(-1)^{w_{H}(v)+1} \widehat{\phi_{u}}(v) .
$$

In the non linear case, the resilient property is not a necessary condition to reduce the bias. The Boolean function of the previous example

$$
f(x)=x_{2}+x_{3}+x_{1} x_{3}+x_{2} x_{3} \bmod 2,
$$

is not resilient, but the output bias is reduced. Indeed, the Walsh coefficients of (001) and (100) are not null, but the sum of both is null.

For example, M. Dichtl proposed a non linear corrector mapping 16 bits to 8 bits such that all $e$ powers up to the fifth are gone in the output bias formula [5]. This corrector was found by exhaustive search and the hardware implementation requires a considerable amount of chip area.

The calculation of syndrome of the non linear $(16,256,6)$ Nordstrom-Robinson code provides a $(16,8,5)$-resilient function [10]. Theorem 4, applied to this function, gives a corrector with a valuation of $\Delta_{u}(e)$ equal to 6 and with a possible implementation for smart-card applications.

\section{$5 \quad$ Bias and Minimal Entropy}

For the evaluation of the random quantity in a binary sequence, the minimal entropy is an appropriate notion for random number generation in cryptography [3. In this part, we prove that if the bias of any non zero linear combination of output bits is bounded, then the minimal entropy of the output can be estimated. Theorem 5 gives the relation between one-dimensional bias $\Delta_{u}(e)$ and multidimensional bias and follows from [1, [2].

Theorem 5. Let $f$ be a function from $\mathbf{F}_{2}^{n}$ to $\mathbf{F}_{2}^{m}$. For all $y \in \mathbf{F}_{2}^{m}$, the multidimensional bias

$$
\left|P(f(x)=y)-2^{-m}\right|
$$

is less or equal than

$$
2 \max _{u \in \mathbf{F}_{2}^{m}}\left|\Delta_{u}\right| .
$$


Definition 3. Let $X$ be a discrete random variable on $\{0,1\}^{n}$. The minimal entropy of $X$ is the maximal number $k$ such that

$$
\forall x \in X, \quad P(X=x) \leq 2^{-k} .
$$

Theorem [5 is a good tool to evaluate minimal entropy of the output. Indeed, we suppose that a $(n, m, t)$-resilient function is used, with an input bias $e$. Then, with Theorems 4 and 5 the bias of any output $m$-tuple is a polynomial of valuation greater than $t+1$. If $e^{t+1}$ is negligible compared to $2^{-m}$, then the minimal entropy of the output is very close to $m$.

With a linear $(n, m, t)$-resilient function and an input bias $e$, we have

$$
P(f(x)=y) \leq 2^{-m}+2^{t+1} e^{t+1},
$$

then the minimal entropy of the output is greater than

$$
m-\log _{2}\left(1+e^{t+1} 2^{m+t+1}\right) .
$$

For example, if $e=1 / 4$, then

$$
P(f(x)=y) \leq 2^{-m}+2^{-(t+1)},
$$

For a linear cyclic code, a syndrome is computed with a modular polynomial reduction, which is realized by using a linear feedback shift register. Lemma 2 explains how getting linear resilient function by calculating a syndrome. Let $C$ a $[n, k, d]$ linear code, $H$ its check matrix and $d^{\prime}$ its dual distance, then the function $x \mapsto H .^{t} x$ is a $\left(n, n-k, d^{\prime}-1\right)$-resilient function.

Let $C$ the $[255,21,111] \mathrm{BCH}$ code, $D$ the $[255,234,6]$ dual code of $C$, with generator polynomial

$$
H(X)=X^{21}+X^{19}+X^{14}+X^{10}+X^{7}+X^{2}+1 .
$$

The input 255 -tuple $\left(m_{1}, \ldots, m_{255}\right)$ is coded by a binary polynomial $m(X)=$ $\sum_{i=1}^{255} m_{i} X^{i}$. Therefore the function $f$ mapping $\mathbf{F}_{2}^{255}$ to $\mathbf{F}_{2}^{21}$, defined by

$$
m(X) \mapsto m(X) \bmod H(X)
$$

is a $(255,21,110)$-resilient function. This polynomial reduction is implemented by a shift register of length 21 with only seven xor gates.

In this case, with an important input bias $e=0.25$, Theorems 3 and 5 give an output bias of :

$$
\forall y \in \mathbf{F}_{2}^{21} \quad\left|P(f(X)=y)-2^{-21}\right| \leq 2^{-111} .
$$

Therefore, the minimal entropy of the output is very close to 21 .

\section{Conclusion}

In this work we present general constructions of good post-processing functions. We have shown that linear correcting codes and resilient functions provide many correctors achieving good bias reduction with variable input sizes. Linear feedback shift register are suitable for an hardware inplementation where the chip area is limited. 


\section{Acknowledgment}

The author would like to thank Philippe Langevin for helpful discussions. The author want also thank to Kaisa Nyberg for useful comments on the paper and for the proof of Theorem 2, which is more elegant than original proof presented at the workshop.

\section{References}

1. Alon, N., Goldreich, O., Hastad, J., Peralta, R.: Simple Constructions of Almost $k$ wise Independent Random Variables. In: IEEE Symposium on Foundations of Computer Science, pp. 544-553., http://citeseer.ist.psu.edu/alon92simple.html

2. Baignères, T., Junod, P., Vaudenay, S.: How far can we go beyond linear cryptanalysis. In: Lee, P.J. (ed.) ASIACRYPT 2004. LNCS, vol. 3329, pp. 432-450. Springer, Heidelberg (2004)

3. Barker, E., Kelsey, J.: Recommendation for random number generation using deterministic random bit generators (revised). NIST Special publication 800-90 (March 2007), http://csrc.nist.gov/publications/nistpubs/800-90/SP80090revised_March2007.pdf

4. Chor, B., Goldreich, O., Hastad, J., Freidmann, J., Rudich, S., Smolensky, R.: The bit extraction problem or t-resilient functions. In: Proc. 26th IEEE Symposium on Foundations of Computer Sciences, pp. 396-407 (1985), http://citeseer.ist.psu.edu/chor85bit.html

5. Dichtl, M.: Bad and good ways of post-processing biased physical random numbers. In: Biryukov, A. (ed.) FSE 2007. LNCS, vol. 4593, pp. 127-152. Springer, Heidelberg (2007)

6. Grassl, M.: Code table: bounds on the parameters of various types of codes, http://www. codestables.de

7. Matsui, M.: Linear cryptanalysis method of DES Cipher. In: Helleseth, T. (ed.) EUROCRYPT 1993. LNCS, vol. 765, pp. 386-397. Springer, Heidelberg (1994)

8. Mac Williams, F.J., Sloane, N.J.A.: The theory of error correcting codes. NorthHolland, Amsterdam (1977)

9. Martin, W.J., Sunar, B., Stinson, D.R.: A provably secure true random number generator with built in tolerance to active attacks. IEEE Transactions on computers 56(1), 109-119 (2007)

10. Stinson, D.R., Massey, J.: An infinite class of counterexamples to a conjecture concerning non linear resilient functions. Journal of cryptology 8(3), 167-173 (1995), http://citeseer.ist.psu.edu/629195.html

11. Xiao, G.: Massey: A spectral Characterization of correlation immune functions. IEEE Transactions on information theory V 34, 569-571 (1988) 